\title{
Impaired Cognitive Flexibility and Working Memory Precedes Depression: A Rat Model to Study Depression
}

\author{
Margarita M. Maramis ${ }^{a} \quad$ Marlina S. Mahajudin ${ }^{a} \quad$ Junaidi Khotib ${ }^{b}$ \\ ${ }^{a}$ Department of Psychiatry, Faculty of Medicine, Airlangga University, Surabaya, Indonesia; \\ ${ }^{b}$ Department of Clinical Pharmacy, Faculty of Pharmacy, Airlangga University, Surabaya, Indonesia
}

\section{Keywords}

Cognitive impairment $\cdot$ Correlation of time $\cdot$ Depression

\begin{abstract}
Introduction: Depressive disorders are the 4th leading cause of health problems and the 2 nd leading cause of burden among all diseases. Almost all depressive disorder patients have cognitive impairments to a certain extend. Studies about cognitive impairments in depression had been conducted, but whether cognitive dysfunctions are the cause or the effect is still not clear. Objectives: To analyze the process of working memory and cognitive flexibility impairments in a rat model of depression. Methods: In this experimental study, chronic unpredictable mild stress (CUMS) was used as a model of depression in 30 rats (Rattus novergicus). Cognitive function was assessed with the Morris water maze and attentional set shifting test. Results: This study found a significant difference on day 21 in working memory ( $p=0.002)$ and cognitive flexibility $(p=0.036)$, which continued to day 41 in working memory $(p=0.001)$ and cognitive flexibility $(p=0.020)$. In the CUMS model of depression, parameters peak on day 41 and reveal parameter changes in weight gain $(p=0.018)$, food intake $(p<0.001)$, changes in food intake $(p=0.001)$, and the sucrose preference ( $p=$
\end{abstract}

karger@karger.com www.karger.com/nps

Karger $\stackrel{\text { ' }}{5}$

GOPEN ACCESS
(C) 2020 The Author(s)

Published by S. Karger AG, Basel

This article is licensed under the Creative Commons AttributionNonCommercial-NoDerivatives 4.0 International License (CC BY NC-ND) (http://www.karger.com/Services/OpenAccessLicense) Usage and distribution for commercial purposes as well as any distribution of modified material requires written permission.
$0.005)$, elevated plus maze ( $p=0.001)$, and light dark box tests $(p=0.020)$. Conclusion: In a rat model of depression, cognitive impairment preceded depression, but it might be caused by anxiety-like behavior that occurred in early stimulation of chronic unpredictable mild stress.

(C) 2020 The Author(s)

Published by S. Karger AG, Basel

\section{Introduction}

Depressive disorder, which affects 151.2 million of the world population, is the 4 th leading disease that causes health problems [1] and the 2nd leading cause of overall disease burden calculated for all ages [2], i.e., $4.3 \%$ of years lost to disability (disability-adjusted life years) [1]. The lifetime prevalence of depression in the adult population is between 15 and $25 \%$, while the point prevalence in a community varies between 5 and $9 \%$ for women and 2 and $3 \%$ for men [3-5]. Cognitive problems affected by major depressive disorder are as high as $85-94 \%$ of the time during depressive episodes and $39-44 \%$ of the time during remissions $[6,7]$.

Cognitive function is the main depiction of major depression [8] with symptoms encompassing affective, cognitive, and somatic functions, anxiety, and psychomotor 
functions [9]. In depressive disorder, cognitive impairment is found in children, adolescents, adults, as well as the elderly [10]. The prevalence of severe cognitive distortion in depressed adolescents is $47.4 \%$, while the rest are less severe [11]. Cognitive impairment in depression was due to cortical atrophy that implicates cognitive function [12], which worsens if not treated [13], stays even after the depression is in remission or recovery and medication is stopped [14-16], and worsens after recurring episodes of depression [8]. Cognitive impairment may disable an individual from having a positive social interaction and may cause various conflicts and self-withdrawal that can be very damaging for the patient's future $[10,17]$. Almost all research on depression focuses on cognitive impairment in depressed individuals, but some questions still remain: Does cognitive impairment occur before depression or as a consequence of being depressed? Does the cortical atrophy that was responsible for cognitive impairment occur before or after depression onset or both? In other words, was it due to cognitive impairment that patients cannot think strategically and flexibly, thus struggle to cope with the problems in their life, and finally suffer from depression, or vice versa?

Cognitive function is very important in processing perception/information from outside and inside oneself, in order that the individual is capable of facing life events and becoming resilient. It is needed to handle depressive feelings and process information, as well as to prevent the risk of depression recurrence [18]. Cognitive functions that are important to humans are working memory and cognitive flexibility. These 2 aspects of cognitive function are essential skills for humans. As a basic and fundamental cognitive process, working memory is the brain system that processes the storage of stimulant and temporary information that needs to be processed and manipulated $[19,20]$. Cognitive flexibility is the ability to adopt or process changes in the cognitive strategy (or behavior) to face new and unexpected conditions from the environment. It is characterized by a learning process, memory and set shifting, an adaptation process, or rearranging of cognitive strategies in response to changes in the environment $[21,22]$.

Cognitive impairment in depression may come in the form of cognitive dysfunction, i.e., cognitive deficits and cognitive bias or distortion [18, 23-25] along with cognitive inflexibility in cognitive function [26]. In terms of more general cognitive aspects, impairments that occur are decreased processing capacity, interpretation, and storage of necessary information [27], and the inability to process information as "neutral" or restrain and delete ir- relevant information which could underlie slowness and attention deficits [28]. In general neurocognitive tests found impairments in various cognitive function domains [29]. The impaired domains include attention, memory, executive function, psychomotor [30], and information processing speed [31], inability to select information, and inability to restrain and delete irrelevant information [28]. Cognitive distortion causes despair and brings up more suicidal thoughts [32], delays recovery, makes the disease chronic, and makes it easier to have depression relapse [15] and maintain depression.

The relationship between cognitive impairment and depression is unclear. Cognitive impairment may cause depression, or, the opposite, depressive disorder may cause cognitive impairment. Therefore, this research aimed to analyze the processes changing in cognition, i.e., working memory and cognitive flexibility, in a rat model of depression received chronic unpredictable mild stress (CUMS). In this model, daily mild stressors, which resemble the stress factors of daily life, are applied. CUMS was the first systematically investigated animal model of depression fulfilling construct validity beside predictive validity (performance in the test predicts performance in the condition being modeled), face validity (phenomenological similarities between the 2), construct validity (has a sound theoretical rationale), and translational potential [33-36]. Although the model was often criticized for a perceived lack of reliability due to the unreliability of the procedure, fact is that this model has been successfully used by hundreds of laboratories worldwide $[37,38]$.

\section{Methods}

Design

Experimental laboratory research was performed using a completely randomized design. Experimental animals used were outbred stock white rats (Rattus norvegicus) meeting the following inclusion criteria: male, 4 months old, weight 175-275 g, healthy condition: marked by shiny and clean fur, shiny eyes, agile movements, and calm demeanor. Exclusion criteria were physical illness, physical disability or wounds on the body, or secretions around the anal canal.

\section{Replication}

The number of animals calculated based on the Federer formula (1955) was 30 rats, which were randomly allocated to one of 5 experimental groups with complete randomization method: 3 treatment groups for observations on the 21st day (P1), 31st day (P2), and 41st day (P3), respectively, and 2 control groups for observations on the 21 st day (K1) and 41st day (K2). The control groups (no CUMS intervention) are placed in a quiet room with the same lighting, temperature, and humidity conditions. 


\section{Depression Model}

For the animal model of depression, CUMS was applied to white rats, which causes depression that resembles the depression in humans. CUMS involves the administration of various unexpected treatments that were given daily and randomly as follows: the animal's tail was tied using a string for $1 \mathrm{~h}$; $30 \mathrm{~min}$ of continuous low-speed treadmill; 10 min of electrical shock for $0.2 \mathrm{~s}$ with 2 -mA current at $470 \mathrm{~V}$ every $2 \mathrm{~s} ; 5 \mathrm{~min}$ of swimming; $60 \mathrm{~min}$ of the animal's tail being pierced for $2.5 \mathrm{~cm} ; 4 \mathrm{~h}$ of exposure to a cat predator accompanied by angry cat sound; $4 \mathrm{~h}$ of isolation in a narrow and dark space; and $4 \mathrm{~h}$ of exposure to an older intruder mouse. These treatments were given to all rat subjects as an animal model of depression $[39,40]$. White rat subjects were given CUMS treatment for 21, 31, and 41 days. Conditions indicating depression used were changes in the following parameters versus baseline parameters of the control groups [41]:

- Increase or decrease in body weight of the treatment group relative to the baseline of the control group ( $\mathrm{K} 1+\mathrm{K} 2$ combined): body weight is the weight of the rat body that was measured weekly using the same scale [42].

- Food intake of the treated group, which may increase or decrease: therefore, it was compared with the control group. Food intake was measured by the number of pellets consumed daily. It was assessed by weighing the pellets that were given daily, which amount to $10 \%$ of animal body weight, subtracted by the leftovers measured at the same time daily. The average food intake was measured daily for the period before treatment (day 0) up to treatment P1 (days 1-21), treatment P2 (days 22-31), and treatment P3 (days 32-41) [42].

- Sucrose preference test (SPT): SPT of the treatment group was compared to the control group. Drink intake was the amount of $1.5 \%$ diluted sucrose in water $(1-2 \%)$ plus normal water that was consumed daily. It was measured by SPT, which is the average amount of sucrose water consumed divided by the total amount of sucrose water and normal water consumed daily. Sucrose water and normal water were given daily $(250 \mathrm{~mL})$. The leftovers were measured daily at the same time, which give the expected direction of change for treated animals [42].

- Elevated plus maze (EPM) test: the amount of time a rat spends in the open space, measured for $15 \mathrm{~min}$ (900 s), which was expected to decrease in the treated group compared to controls $[43,44]$.

- Light dark box (LDB) or white dark box test: the length of time a rat spends in open space during a 15-min period, which was expected to decrease in the treatment group compared to the control group [41].

\section{Cognitive Test}

Cognitive impairment is an abnormal condition of the cognitive function which can be reversible or irreversible, which may be caused by abnormality in function and/or structure. This condition does not indicate a diagnostic entity. For measuring cognitive function (in our case working memory and cognitive flexibility), the learning intelligence cognition hierarchy (LICH) was used: LICH level 4 (chaining: learning sequences of stimulus-response learning) in the form of Morris water maze (MWM) and $\mathrm{LICH}$ level 5 (multiple discrimination learning: concurrent discrimination learning or learning set formation) in the form of set shifting [45-47]. Working memory, a process of simultaneous and temporary information storage, is needed for processing and manipula- tion $[19,20]$. This research examined spatial working memory that was measured using MWM that assessed the length of time a rat was able to be in the pool quadrant with an elevated platform in $120 \mathrm{~s}$ [46]. Cognitive flexibility, the ability to adapt or the process to change a cognitive strategy (or behavior) to deal with a new and unexpected condition from the environment [21, 22], was measured by the attentional set shifting test/task (AST), which was the number of times the test animal was able to locate food that was associated with smell or other media, and the animal needed to achieve the correct response criteria for 6 consecutive times at each stage known as trial to criterion [47].

The MWM is a gold standard test used to study spatial memory and learning, developed by Richard G. Morris (1984). Rats are placed in a pool of water that is colored opaque with powdered nonfat milk or nontoxic paint, where they must swim to a hidden escape platform. The rats cannot see the platform and cannot rely on scent to find the escape route. Instead, they must rely on external/extra maze cues. The longer the time the rats took to reach the elevated platform quadrant, the poorer the spatial memory and learning $[46,48]$.

\section{Attentional Set Shifting Test/Task}

Rats are initially allowed to dig in sawdust-filled bowls containing a food reward. Once the rats are reliably finding the rewards, the rats learn simple discriminations where the bowls differ in only one aspect or perceptual dimension (e.g., by digging medium: shredded paper compared to polystyrene pieces). Rats are deemed to have learned a discrimination when they reach a criterion performance of 6 consecutively correct trials. The following day, rats undertake a series of seven 2-choice discriminations designed to measure acquisition, reversal learning, and the cost of shifting set - each with a learning criterion of 6 consecutive correct choices. The task starts with a simple discrimination between either 2 odors in sawdust or 2 digging media with no added odor. Then the complementary, but task-irrelevant, stimulus dimension is added to form the compound discrimination (CD). In the first reversal (REV1), the outcome contingencies of the CD are swapped such that the correct response for the $\mathrm{CD}$ is now incorrect, and vice versa. Then new compound stimuli, differing again according to odor and digging medium, are introduced for the intradimensional shift acquisition. The relevant (that is, reward-predicting) dimension remains congruent with prior stages. After that follows a second reversal stage (REV2). In the extradimensional shift stage, the exemplars are changed again, but now the relevant dimension is incongruent with the prior stages: stimuli in a previously irrelevant dimension predict reward for the first time. This series of discriminations - which is almost always conducted in a single session on 1 day - ends with a final reversal (REV3) $[49,50]$.

\section{Results}

\section{Depression Model}

CUMS treatment effects were evaluated by univariate analyses of variance (ANOVA) comparing the 5 groups with respect to depression-associated variables before treatment at $\mathrm{t} 0$; $t$ tests were computed for comparisons between each treatment and the respective control group. 


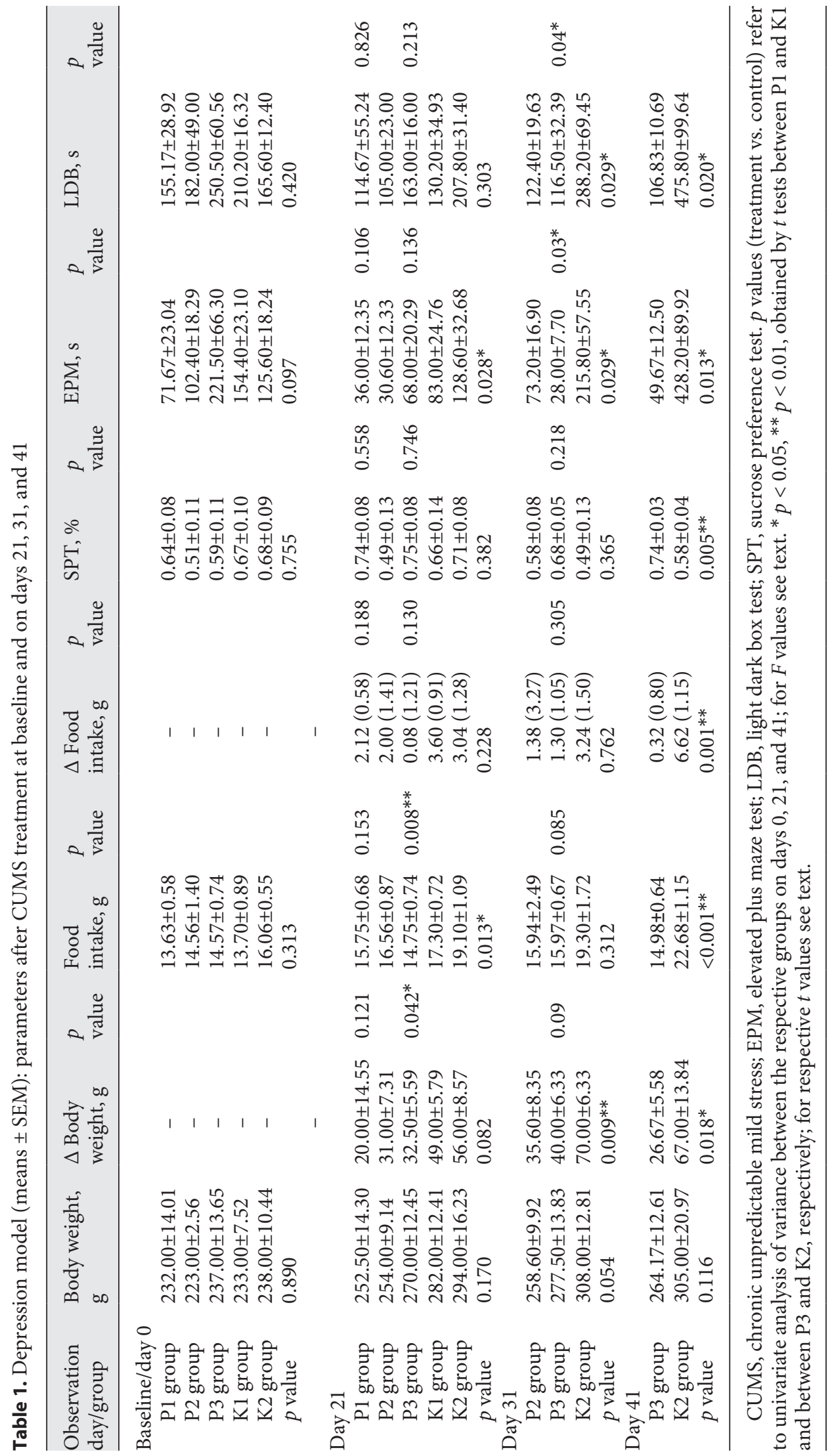




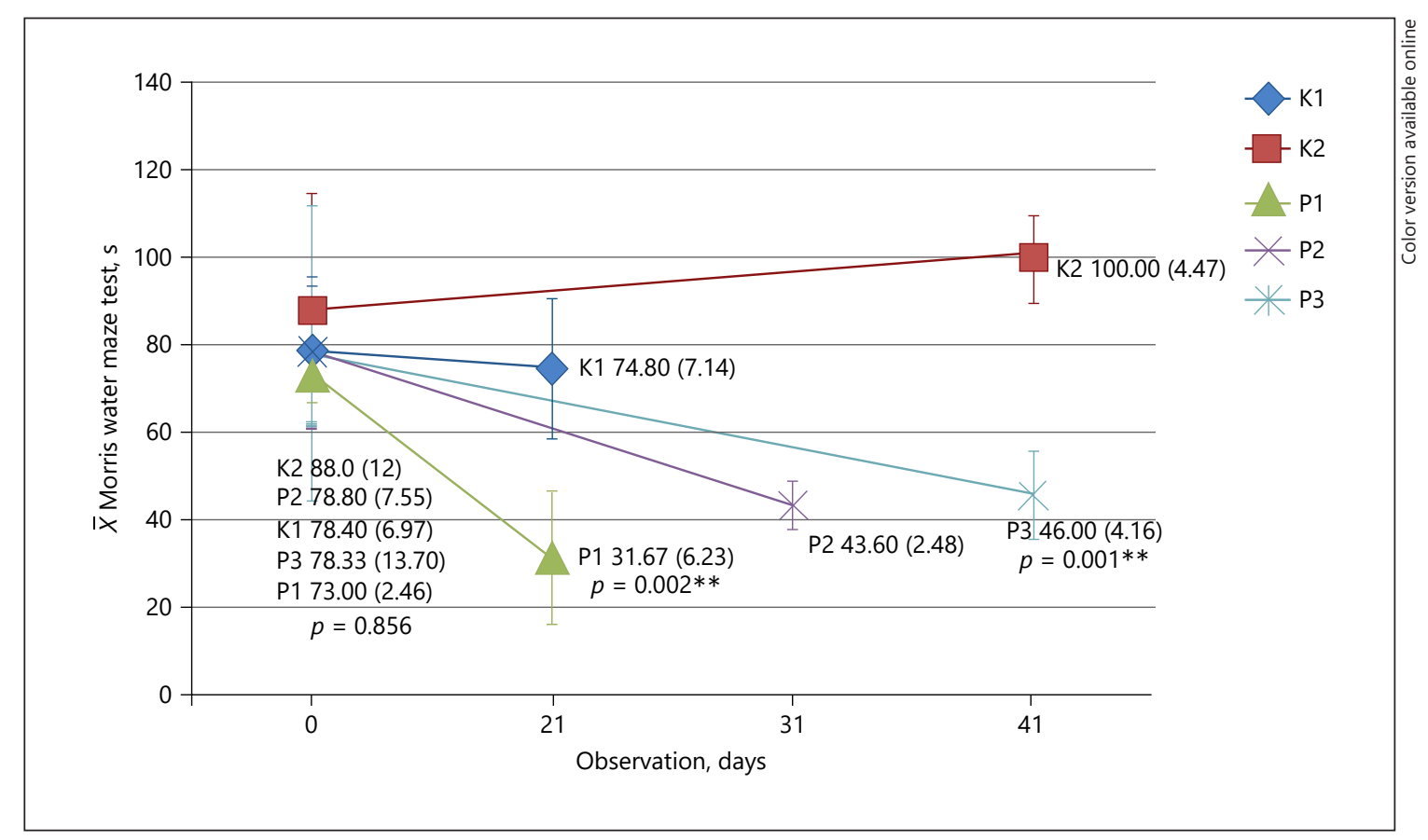

Fig. 1. MWM test results at baseline and on days 21,31, and 41. Means and SEM; significance refers to $F$ value of analysis of variance at $t 0(F=0.326$; $\mathrm{df} 1=4 ; \mathrm{df} 2=14.039 ; p=0.856)$ and to $t$ tests between treated and control groups at the respective time points, i.e., day 21 : P1 vs. K1 $(t=4.57 ; \mathrm{df}=9 ;=0.002)$ and day 41 : P3 vs. K2 $(t=$ $8.82 ; \mathrm{df}=9 ; p=0.001)$, no comparisons for P2.

No difference was observed between the control and treatment group on day 0 in body weight $(F=0.277$; df $1=4$; df $2=22 ; p=0.890)$; food intake $(F=1.266$; df $1=4 ;$ df $2=22 ; p=0.313)$; SPT $(F=0.474 ;$ df $1=4$; df $2=22 ; p=0.755), \operatorname{EPM}(F=2.746 ; \mathrm{df} 1=4 ; \mathrm{df} 2=8.89$; $p=0.097)$, and LDB $(F=1.054$; df $1=4$; df $2=12.3 ; p=$ 0.420 ) and on day 21 between each treatment and control group for $\mathrm{P} 1-\mathrm{K} 1$ and $\mathrm{P} 3-\mathrm{K} 2$, respectively, on change in body weight $(t=1.715 ; \mathrm{df}=9 ; p=0.121$; and $t=2.373$; $\mathrm{df}=9 ; p=0.042)$, food intake $(t=1.562 ; \mathrm{df}=9 ; p=0.153$; and $t=3.682 ; \mathrm{df}=9 ; p=0.008)$, decrease in the change in food intake $(t=1.425 ; \mathrm{df}=9 ; p=0.188$; and $t=1.666$; $\mathrm{df}=9 ; p=0.130)$, increase in SPT $(t=-0.608 ; \mathrm{df}=9 ; p=$ 0.558 ; and $t=-0.334 ; \mathrm{df}=9 ; p=0.746), \mathrm{EPM}(t=1.795$; $\mathrm{df}=9 ; p=0.106$; and $t=1.635 ; \mathrm{df}=9 ; p=0.136)$, and LDB $(t=0.226 ; \mathrm{df}=9 ; p=0.826$; and $t=1.341 ; \mathrm{df}=9 ; p=0.213)$. From CUMS treatment, significant differences in all measured depression model parameters were observed on day 41 of treatment between the control and treatment group regarding change in body weight $(t=2.895 ; \mathrm{df}=9 ; p=$ $0.018)$, decrease in food intake $(t=6.124 ; \mathrm{df}=9 ; p<$ $0.001)$, change in food intake $(t=4.632 ; \mathrm{df}=9 ; p=0.001)$, increase in SPT $(t=-3.741 ; \mathrm{df}=9 ; p=0.005)$, EPM $(t=$
4.169; $\mathrm{df}=4.16 ; p=0.013)$, and $\operatorname{LDB}(t=3.682 ; \mathrm{df}=4.09 ;$ $p=0.020)$. This means that complete depression symptoms occurred on day 41 (Table 1).

\section{Cognitive Functions}

The same procedures as described above were applied to all variables associated with cognitive impairment (day 21: P1 versus K1; day 41: P3 versus K2, no comparisons for $\mathrm{P} 2$ ). The following results were obtained:

- Means and standard error of the means (SEM) for each group were: $73.00(2.46)$ at $\mathrm{P} 1,78.80(7.55)$ at $\mathrm{P} 2,78.33$ (13.70) at P3, $78.40(6.97)$ at K1, and 88.00 (12) at K2. No difference in MWM $(F=0.326$; df $1=4$; df $2=$ $14.039 ; p=0.856)$ and $\operatorname{AST}(F=3.445 ; \mathrm{df} 1=4$; df $2=$ $6.953 ; p=0.074$ ) results were found at baseline/t0 (day 0 ) measurements among groups (Fig. 1). Means and SEM for each group were $79.40(0.24)$ at K1, 86.67 (3.48) at $\mathrm{P} 1,79.20(0.58)$ at $\mathrm{P} 2,80.60(0.93)$ at $\mathrm{K} 2$, and 79.67 (1.09) at P3. No difference in AST $(F=3.445 ; \mathrm{df}$ $1=4$; $\mathrm{df} 2=6.953 ; p=0.074)$ results was found at baseline/t0 (day 0) measurements among groups (Fig. 2).

- Means and SEM were 31.67 (6.23) at P1, 74.80 (7.14) at K1, 46 (4.16) at P3 and 100 (4.47) at K2. A significant 


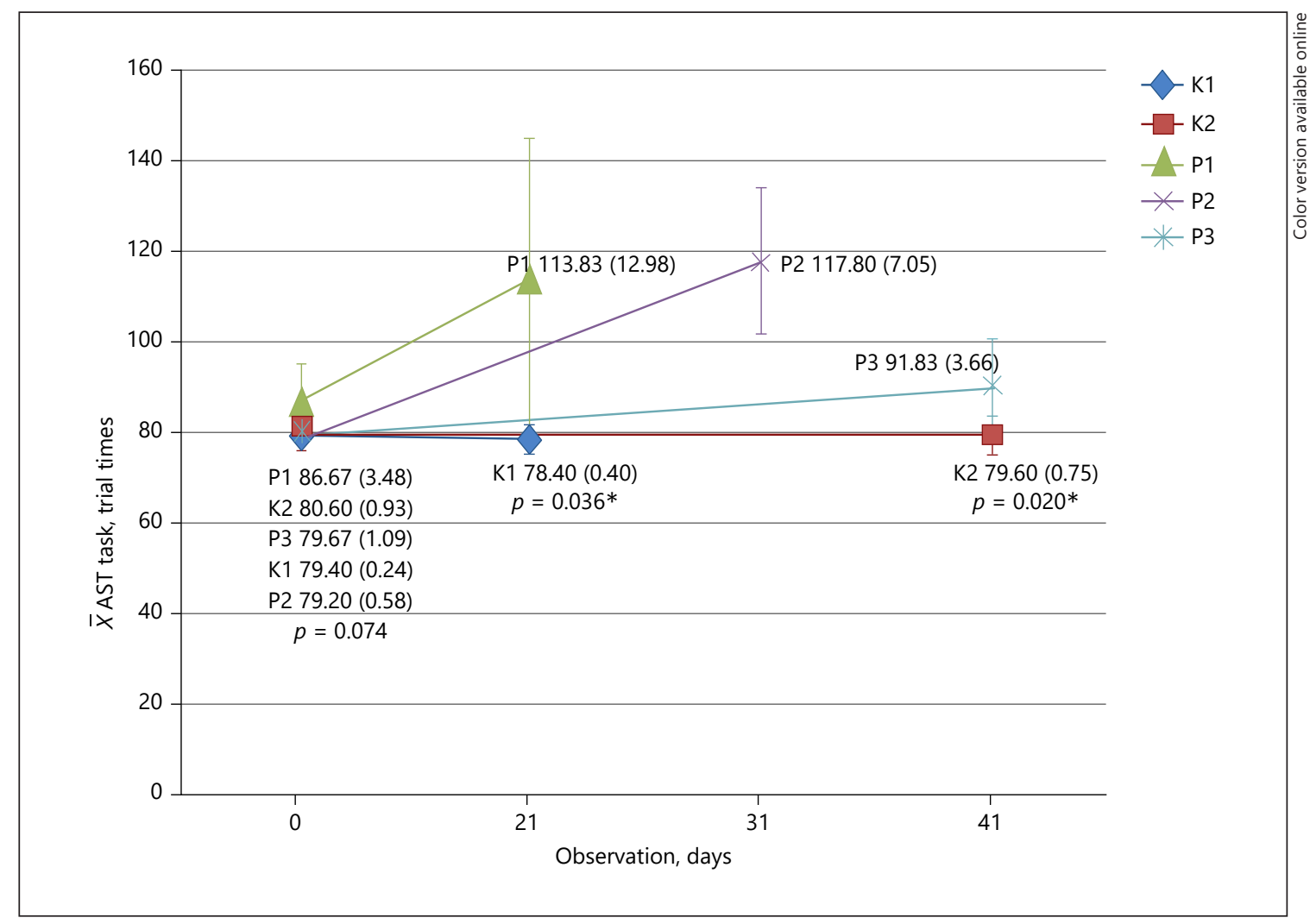

Fig. 2. AST results at baseline and on days 21,31, and 41. Means and SEM; significance refers to $F$ value of analysis of variance at $t 0(F=3.445 ; \mathrm{df} 1=4 ; \mathrm{df} 2=6.953 ; p=0.074)$ and to $t$ tests between treated and control groups at the respective time points, i.e., day 21 : $\mathrm{P} 1$ vs. $\mathrm{K} 1(t=-2.47 ; \mathrm{df}=9 ; p=0.036)$ and day 41 : $\mathrm{P} 3$ vs. $\mathrm{K} 2(t=-3.27$; $\mathrm{df}=5.41 ; p=0.020)$, no comparisons for P2.

decrease was observed comparing the treatment groups with the control groups on day 21 (P1 vs. K1: $t=4.57 ; \mathrm{df}=9 ; p=0.002$ ) and day 41 (P3 vs. K2: $t=$ $8.82 ; \mathrm{df}=9 ; p=0.001)$. A decrease in MWM test results suggests a decrease in memory function in the treatment group compared with the control group since day 21 and continued up to day 41 (Fig. 1).

- Means and SEM were 113.83 (12.98) at P1, 78.40 $(0.40)$ at $\mathrm{K} 1$ on day 21, and on day 4191.83 (3.66) at $\mathrm{P} 3$, and $79.60(0.75)$ at K2. A significant increase was observed in the treatment group in comparison to the control group on day 21 (P1 vs. K1: $t=-2.47 ; \mathrm{df}=9$; $p=0.036)$ and day 41 (P3 vs. K2: $t=-3.27$; $\mathrm{df}=5.41$; $p=0.020)$. The increase in AST test results indicated that in order to find the reward in the form of food that was associated with a certain smell and media, the rat made more mistakes, so that it needed to be repeated several times. This results suggest that there was a decrease in cognitive flexibility in the treatment group compared to the control group since day 21 that continued up to day 41 (Fig. 2).

\section{Discussion}

Increases in food intake, body weight, and drink intake occurred in the early stages up to day 31 , after which a decline occurred in the CUMS-treated group versus the control group that continued to experience increases in food intake and body weight up to day 41 , when food intake and body weight stabilized. The presence of a stimulation that continuously increases body response, which occurs continuously, will at some point cause a decline as stated in a theory by Selye $[51,52]$. To deal with an external stimulus, humans require energy that needs to be achieved by the body by food and drink intake to be able to balance or adapt to the stressors. Since the stressors or stimuli that were given daily were of a mild degree, this 
would lead to an increase in body weight. If the stimulus was continued, the metabolic system that was continuously stimulated will become fatigued and will decrease its response activity. The food intake that experienced an increase in the beginning will decline along with body weight. Despite that, several controversial reports found an immediate decline in body weight and sucrose water intake after experiencing various stressors. For example, a decline in SPT under depressed condition [42] or an increase in SPT compared to control $[53,54]$. These conditions differ individually, and in rats they may be caused by different rat strains and different exposure times. Application of daily mild stressors do not cause shock and appear to inhibit shock response during the alarm reaction phase according to the Selye theory $[52,55]$. Mild stressors do not suppress response as shown in the table and figures, therefore, no acute decline in the body reaction occurred.

To fulfill depression syndrome model parameters, 3141 days were requested, and in this research, compared to control, significant changes in all depression model parameters occurred on day 41 , marked by changes in body weight, amount of food and drink intake, EPM, and LDB. The 5 parameters in the rat model of depression did not show significant changes relative to control simultaneously. This indicated that each biological process that underlies behavior in individuals has a different rate of change that is seen as different irritation or distress, which in humans would manifest as different complaints that do not occur simultaneously. The body has various systems that work according to natural processes. A stimulation or stressor that affects several systems may cause imbalance. Each system may reciprocally affect each other, which causes changes in the form of deceleration or acceleration of subsequent cascades [56, 57]. With symptoms that greatly vary individually, some symptoms may be noticed earlier before the others, which may cause individuals to not consider them as an urgent matter that needs resolving. Only when symptoms have progressed in severity and multiply does the individual seek help.

The 2 cognitive tests showed that CUMS as depression model did provide depressive effects for all parameters on day 41: a decreasing effect in spatial working memory and cognitive flexibility since day 21 . Other research, where CUMS stressor treatment was given for 10 days, also had the same findings, and a decrease in the platform finding strategy in the MWM test was observed with an increase in the length of the route traveled to reach the platform due to a decrease in memory [58]. Working memory capability and cognitive flexibility decreased, and thus the capacities to process information and think to acquire alternatives became limited. Cognitive function that is impaired in depressive disorder is very harmful since cognitive functions are required to handle everyday life. If an impairment occurs, mistakes in decision making may happen, which give rise to suicidal thoughts [32], result in the failure to recognize the individual's state that is in need of help [59], prolong recovery, proceed into a chronic condition, enable a depression relapse to occur $[15,18]$, and eventually lead to low quality of life even if the depression is in remission [60]. This suggests that cognitive function impairment, in this case working memory function and cognitive flexibility, occurred before depressive disorder parameters presented completely.

Continuous stimulation may surpass the limits of cognitive ability that cause stress and eventually depression. Here, the proposed mechanism of depression was a stress condition with continuous stimuli, which led to initial anxiety-like behavior and impairment in cognitive function and, as the stressors continuously presented, finally caused depression and continuously impaired cognitive function. Indeed, most cognitive theories propose vulnerability-stress hypotheses that posit that the onset of this disorder is due to the interaction of a psychological vulnerability (e.g., certain cognitions or particular ways of processing information) and a precipitating stressor (e.g., a negative life event or some other environmental factor) [18].

Analyzed further, cognitive impairment occurred on day 21, while depression symptoms from CUMS treatment were only completely displayed on day 41 . Despite that, some distressed behavior symptoms were already observed on day 21 compared with day 0 in the form of confused behavior, instability, and behaviors such as circling, being afraid, not concentrating, shaking, and easily getting startled, sensitivity to noise, odors, and movements, and aggressive behavior. On day 41, the distressed behavior was even more apparent as rats are moving more slowly and took a long time to complete the tests, made more mistakes, were stopping and had to be pushed, and were even staying still. These behaviors resemble anhedonia symptoms in humans. This cognitive impairment condition was also found in patients with depressive disorder that experienced psychic and cognitive symptoms but were ignoring them. Only after the symptoms worsened and the patient was unable to function daily do they seek help. For that, early detection of impairments in cognitive function and depressive disorder is necessary as a preventative measure $[33,35,36]$. In another study, behaviors resembling anxiety and cognitive 
deficits were observed in rats given CUMS treatment for 14 days [61]. Thus, the observed cognitive impairment that occurred on day 21 might either be caused by stressors or by anxiety-like behavior effects; although that this was not universally observed, the anxiogenic effects frequently occur in the CUMS model [38]. These findings also raise the question of whether depression should be termed as a mood or cognitive disorder. It seems that cognitive disorders are a core pathological symptom of depression and should not be considered merely secondary to it, although most of the current treatments focus on mood dysregulation. Cognitive symptoms should, therefore, be regarded as a partially independent dimension of the major depressive disorder and an important target of any treatment that is initiated $[62,63]$. Cognitive deficits predicting a major depressive disorder likely represent deleterious effects of subclinical depression symptoms on performance rather than premorbid risk factors for the disorder [64].

Even though the result is like above, they may turn out different, if determination of stress parameters would be different. To elucidate further details on the process and correlation between cognitive function and depression, further study is required with prolonged follow-ups focusing on cognitive function after CUMS stressor was stopped. This study concludes that cognitive impairment occurred before depression in a rat model of depression; however, it might be caused by anxiety-like behavior that occurred in early stimulation of chronic unpredictable mild stress.

\section{Acknowledgment}

We are grateful to the late Prof. J.M. Harjanto, MD, AIFM, $\mathrm{PhD}$, the late Prof. Aboe Amar Yusuf, MD, PhD, Susanti Machmud, S.Kom., Sri Hariastuti, Amd., Endah Sujani, S.Si., Tania Ardiani Saleh Hariadi, dra., MS., Atika, S.Si., M.Kes., Tuti Herwini, MD, psychiatrist, PhD, Gladdy Waworuntu, MD, MS, Sr. Adriana Endang, MP, and ALMA, Djuhani, Niniek, Emmy, Yenny, Ali Wardhana,Andi, Danang, Sofyan, Penny (all MD), and Purwandi, Rudi, Maria, Mistam, Trimo, and Risye for their help with this study.

\section{Statement of Ethics}

Ethical clearance was obtained from the Airlangga University Animal Care and Use Committee (No. 229-KE).

\section{Disclosure Statement}

The author state that they have no conflict of interest to declare.

\section{References}

1 Conner M. When are antidepressants better than psychotherapy. 2006. Available from: OregonCounseling.org.

2 Ledford $\mathrm{H}$. If depression were cancer. Nature. 2014;515:182-4.

3 American Psychiatric Association (APA). Diagnostic and statistical manual of mental disorders. 4th ed., text revised. Washington (DC): American Psychiatric Association; 2000.

4 Tasman A, Kay J. Essentials of psychiatry. Chicester: Wiley; 2006.

5 Maletic V, Robinson M, Oakes T, Iyengar S, Ball SG, Russell J. Neurobiology of depression: an integrated view of key findings. Int J Clin Pract. 2007 Dec;61(12):2030-40.

6 Listunova L, Roth C, Bartolovic M, Kienzle J, Bach C, Weisbrod M, et al. Cognitive impairment along the course of depression: nonpharmacological treatment options. Psychopathology. 2018;51(5):295-305.

7 Perini G, Cotta Ramusino M, Sinforiani E, Bernini S, Petrachi R, Costa A. Cognitive impairment in depression: recent advances and novel treatments. Neuropsychiatr Dis Treat. 2019 May;15:1249-58.
8 Monzón S, Gili M, Vives M, Serrano MJ, Bauza N, Molina R, et al. Melancholic versus nonmelancholic depression: Differences on cognitive function. A longitudinal study protocol. BMC Psychiatry. 2010 Jun;10:48.

9 Mayou RA, Sharpe MN, Carson A. Depression in medical practice. ABC of psychological medicine. London: BMJ Publishing Group; 2003.

10 Thomas AJ, Gallagher P, Robinson LJ, Porter RJ, Young AH, Ferrier IN, et al. A comparison of neurocognitive impairment in younger and older adults with major depression. Psychol Med. 2009 May;39(5):725-33.

11 Marton P, Kutcher S. The prevalence of cognitive distortion in depressed adolescents. J Psychiatry Neurosci. 1995 Jan;20(1):33-8.

12 Harvey PD. Cognitive Impairments in Major Depression and Bipolar Disorders. Psychiatry (Edgmont). 2007;4(1):12-14.

13 Gualtieri CT, Johnson LG, Benedict KB. Neurocognition in depression: patients on and off medication versus healthy comparison subjects. J Neuropsychiatry Clin Neurosci. 2006; 18(2):217-25.
14 Neu P, Bajbouj M, Schilling A, Godemann F, Berman RM, Schlattmann P. Cognitive function over the treatment course of depression in middle-aged patients: correlation with brain MRI signal hyperintensities. J Psychiatr Res. 2005 Mar;39(2):129-35.

15 Airaksinen E. Cognitive Functions in Depression and Anxiety Disorders Findings from a population-based study. 2006.

16 Reppermund S, Zihl J, Lucae S, Horstmann S, Kloiber S, Holsboer F, et al. Persistent cognitive impairment in depression: the role of psychopathology and altered hypothalamic-pituitary-adrenocortical (HPA) system regulation. Biol Psychiatry. 2007 Sep;62(5):400-6.

17 Haley GM, Fine S, Marriage K, Moretti MM Freeman RJ. Cognitive bias and depression in psychiatrically disturbed children and adolescents. J Consult Clin Psychol. 1985 Aug;53(4): $535-7$.

18 Gotlib IH, Joormann J. Cognition and depression: current status and future directions. Annu Rev Clin Psychol. 2010;6(6):285-312.

19 Baddeley A. Working Memory Components of Working Memory The Slave Systems of Working Memory Individual Differences in Working Memory. Science. 1992;255:556-9. 
20 Melby-Lervåg M, Hulme C. Is working memory training effective? A meta-analytic review. Dev Psychol. 2013 Feb;49(2):270-91.

21 Evers EA, van der Veen FM, Fekkes D, Jolles J. Serotonin and cognitive flexibility: neuroimaging studies into the effect of acute tryptophan depletion in healthy volunteers. Curr Med Chem. 2007;14(28):2989-95.

22 Zhang H, Kranzler HR, Poling J, Gruen JR, Gelernter J. Cognitive flexibility is associated with KIBRA variant and modulated by recent tobacco use. Neuropsychopharmacology. 2009 Nov;34(12):2508-16.

23 Teasdale JD. Change in Cognition during Depression-Psychopathological Implications: Discussion Paper. J R Soc Med. 1983 Dec;76(12):1038-44.

24 Beck AT. The evolution of the cognitive model of depression and its neurobiological correlates. Am J Psychiatry. 2008 Aug;165(8): 969-77.

25 Clark L, Chamberlain SR, Sahakian BJ. Neurocognitive mechanisms in depression: implications for treatment. Annu Rev Neurosci. 2009;32(1):57-74.

26 Deveney CM, Deldin PJ. A preliminary investigation of cognitive flexibility for emotional information in major depressive disorder and non-psychiatric controls. Emotion. 2006 Aug; 6(3):429-37.

27 Davis S. Cognitive processes in depression. J Clin Psychol. 1982 Jan;38(1):125-9.

28 Gohier B, Ferracci L, Surguladze SA, Lawrence E, El Hage W, Kefi MZ, et al. Cognitive inhibition and working memory in unipolar depression. J Affect Disord. 2009 Jul;116(1-2): $100-5$.

29 Elliott R, Rubinsztein JS, Sahakian BJ, Dolan RJ. The neural basis of mood-congruent processing biases in depression. Arch Gen Psychiatry. 2002 Jul;59(7):597-604.

30 Huang CL. The Value of Patient-administered Depression Rating Scale in Detecting Cognitive Deficits in Depressed Patients. J Clin Med Res. 2010 Feb;2(1):27-33.

31 McDermott LM, Ebmeier KP. A meta-analysis of depression severity and cognitive function. J Affect Disord. 2009 Dec;119(1-3):1-8.

32 Beevers CG, Miller IW. Perfectionism, cognitive bias, and hopelessness as prospective predictors of suicidal ideation. Suicide Life Threat Behav. 2004;34(2):126-37.

33 Willner P. Chronic mild stress (CMS) revisited: consistency and behavioural-neurobiological concordance in the effects of CMS. Neuropsychobiology. 2005;52(2):90-110.

34 Willner P. The validity of animal models of depression. Psychopharmacology (Berl). 1984;83(1):1-16.

35 Willner P. Validity, reliability and utility of the chronic mild stress model of depression: a 10-year review and evaluation. Psychopharmacology (Berl). 1997 Dec;134(4):319-29.
36 Duman $\mathrm{CH}$. Models of depression. Vitam Horm. 2010;82(10):1-21.

37 Willner P. The chronic mild stress (CMS) model of depression: History, evaluation and usage. Neurobiol Stress. 2016 Aug;6:78-93.

38 Willner P. Reliability of the chronic mild stress model of depression: A user survey. Neurobiol Stress. 2016 Aug;6:68-77.

39 Forbes NF, Stewart CA, Matthews K, Reid IC Chronic mild stress and sucrose consumption: validity as a model of depression. Physiol Behav. 1996 Dec;60(6):1481-4.

40 Krishnan V, Nestler EJ. Linking molecules to mood: new insight into the biology of depression. Am J Psychiatry. 2010 Nov; 167(11): 1305-20.

41 Bourin M, Hascoët M. The mouse light/dark box test. Eur J Pharmacol. 2003 Feb;463(1-3): 55-65.

42 Willner P, Towell A, Sampson D, Sophokleous S, Muscat R. Reduction of sucrose preference by chronic unpredictable mild stress, and its restoration by a tricyclic antidepressant. Psychopharmacology (Berl). 1987;93(3): 358-64.

43 Handley SL, Mithani S. Effects of alpha-adrenoceptor agonists and antagonists in a mazeexploration model of 'fear'-motivated behaviour. Naunyn Schmiedebergs Arch Pharmacol. 1984 Aug;327(1):1-5.

44 Walf AA, Frye CA. The use of the elevated plus maze as an assay of anxiety-related behavior in rodents. Nat Protoc. 2007;2(2): 322-8.

45 Bailey AM, McDaniel WF, Thomas RK. Approaches to the study of higher cognitive functions related to creativity in nonhuman animals. Methods. 2007 May;42(1):3-11.

46 D'Hooge R, De Deyn PP. Applications of the Morris water maze in the study of learning and memory. Brain Res Brain Res Rev. 2001 Aug;36(1):60-90

47 Lapiz-Bluhm MD, Bondi CO, Doyen J, Rodriguez GA, Bédard-Arana T, Morilak DA. Behavioural assays to model cognitive and affective dimensions of depression and anxiety in rats. J Neuroendocrinol. 2008 Oct;20(10): 1115-37.

48 Nunez J. Morris water maze experiment. J Vis Exp. 2008 Sep;(19):12-3.

49 Heisler JM, Morales J, Donegan JJ, Jett JD, Redus L, O'Connor JC. The attentional set shifting task: a measure of cognitive flexibility in mice. J Vis Exp. 2015 Feb;(96):2-7.

50 Zhang Y, Shao F, Wang Q, Xie X, Wang W. Neuroplastic Correlates in the mPFC Underlying the Impairment of Stress-Coping Ability and Cognitive Flexibility in Adult Rats Exposed to Chronic Mild Stress during Adolescence. Neural Plast. 2017;2017:9382797.
51 Selye $\mathrm{H}$. The general adaptation syndrome and the diseases of adaptation. J Allergy. 1946; 17(4):231-47.

52 Taylor S, Sirois F. Health Psychology. 2nd Canadian ed. New York: McGraw-Hill Ryerson 2012.

53 Pothion S, Bizot JC, Trovero F, Belzung C. Strain differences in sucrose preference and in the consequences of unpredictable chronic mild stress. Behav Brain Res. 2004 Nov; 155(1):135-46.

54 Strekalova T, Spanagel R, Bartsch D, Henn FA, Gass P. Stress-induced anhedonia in mice is associated with deficits in forced swimming and exploration. Neuropsychopharmacology. 2004 Nov;29(11):2007-17.

55 Pothion S, Bizot JC, Trovero F, Belzung C. Strain differences in sucrose preference and in the consequences of unpredictable chronic mild stress. Behav Brain Res. 2004 Nov; 155(1):135-46.

56 Black PH, Garbutt LD. Stress, inflammation and cardiovascular disease. J Psychosom Res. 2002 Jan;52(1):1-23.

57 Lee S, Kang BM, Shin MK, Min J, Heo C, Lee $\mathrm{Y}$, et al. Chronic stress decreases cerebrovascular responses during rat hindlimb electrical stimulation. Front Neurosci. 2015 Dec;9:462.

58 Gouirand AM, Matuszewich L. The effects of chronic unpredictable stress on male rats in the water maze. Physiol Behav. 2005 Sep; 86(1-2):21-31.

59 Dunlop BW, Dunlop AL. Counseling via analogy: improving patient adherence in major depressive disorder. Prim Care Companion J Clin Psychiatry. 2005;7(6):300-3.

60 Angermeyer MC, Holzinger A, Matschinger $\mathrm{H}$, Stengler-Wenzke K. Depression and quality of life: results of a follow-up study. Int $J$ Soc Psychiatry. 2002 Sep;48(3):189-99.

61 Bondi CO, Rodriguez G, Gould GG, Frazer A, Morilak DA. Chronic Unpredictable Stress Induces a Cognitive Deficit and Anxiety-Like Behavior in Rats that is Prevented by Chronic Antidepressant Drug Treatment. Neuropsychopharmacology. 2008 Jan;33(2):320-31.

62 Miskowiak KW, Ott CV, Petersen JZ, Kessing LV. Systematic review of randomized controlled trials of candidate treatments for cognitive impairment in depression and methodological challenges in the field. Eur Neuropsychopharmacol. 2016 Dec;26(12):1845-67.

63 Planchez B, Surget A, Belzung C. Animal models of major depression: drawbacks and challenges. J Neural Transm (Vienna). 2019 Nov;126(11):1383-408.

64 Scult MA, Paulli AR, Mazure ES, Moffitt TE, Hariri AR, Strauman TJ. The association between cognitive function and subsequent depression: a systematic review and meta-analysis. Psychol Med. 2017 Jan;47(1):1-17. 